\title{
GLOBAL STABILITY AND PERSISTENCE IN DIFFUSIVE FOOD CHAINS
}

\author{
YANG KUANG ${ }^{1}$
}

(Received 20 September, 1995; revised 4 February, 2001)

\begin{abstract}
In this paper, the results of Freedman and So [13] on global stability and persistence of simple food chains are extended to general diffusive food chains. For global stability of the unique homogeneous positive steady state, our approach involves an application of the invariance principle of reaction-diffusion equations and the construction of a Liapunov functional. For persistence, we use the dynamical system results of Dunbar et al. [11] and Hutson and Moran [29].
\end{abstract}

\section{Introduction}

By a food chain of length $n, n \geq 2$, we mean a closed ecosystem (no immigration and emigration) which contains $n$ interacting populations forming $n$ trophic levels, such that each population except the lowest eats the ones on the lower trophic levels. In the following we simply call it a food chain. The other standard name for it is a food web.

The study of food chains dates back to 1926 when Volterra [49] first introduced the so-called Lotka-Volterra predator-prey system, which is a food chain of length two. A large number of food-chain models that have appeared since are of LotkaVolterra type, that is, the per capita growth rates are linear functions. For example, models considered in $[12,21,25,28,31,47,48]$ include the Lotka-Volterra food chain as special cases. Most discussions of more general food chains have been restricted to chains of length two or three (see $[7,12,14-17,45]$ ). For general food chains of arbitrary length, the reader is referred to $[8,9,13,20]$.

Global stability of a feasible steady state or limit cycle is certainly very desirable (see [6]) in real ecosystems. However, it is generally difficult to establish. In fact, even for predator-prey systems such a task is far from trivial (see $[7,22,32,33]$ ).

\footnotetext{
'Department of Mathematics, Arizona State University, Tempe, AZ 85287, USA.

e-mail: kuang@asu.edu

(C) Australian Mathematical Society 2001, Serial-fee code 0334-2700/01
} 
Although there have been results for $n$-dimensional systems, they are valid only for Lotka-Volterra type food chains (see, for example, $[25,28,47]$ ). The only exception is the recent work of Freedman and So [13].

One of the most fundamental questions in mathematical biology concerns the long term survival of each component. This is equivalent to the persistence analysis of the related mathematical models. Persistence has been defined by various authors in a variety of different contexts. If the model consists of only ordinary differential equations, the following definitions are well adopted in the literature (see $[4,5])$ : A vector of $x(t)=\left[x_{1}(t), \ldots, x_{n}(t)\right]$ is said to be weakly persistent if for each component $x_{i}(t), \lim \sup _{t \rightarrow+\infty} x_{i}(t)>0$ and it is said to be strongly persistent if, for each component $x_{i}(t), \liminf _{t \rightarrow+\infty} x_{i}(t)>0$. A system of ordinary differential equations is said to be uniformly persistent if there exists a $\sigma>0$ such that for each component $x_{i}(t), \liminf _{t \rightarrow+\infty} x_{i}(t) \geq \sigma>0$ for all $X=\left[x_{1}(t), \ldots, x_{n}(t)\right] \in$ Int $R_{+}^{n}$. The other analogous terms used in the literature are cooperativeness [28], permanence [30] and permanent coexistence [31]. Similar terms also exist for reaction-diffusion systems (see, for example, [11,29]) and for delay differentiation systems (see [3]). More general and abstract (in terms of semigroup theory) definitions of persistence are given in [24]. Clearly, global stability of a steady state or a limit cycle is much more appealing than persistence in an ecosystem. Intuitively, one may think persistence is easy to establish. Unfortunately, this is not true in the sense that establishing uniform persistence for an $\boldsymbol{n}$-dimensional system is generally just as hard as (if not harder) than showing the global stability of an $(n-1)$-dimensional system (see $[4,13,30]$ ).

This work is motivated by that of Freedman and So [13]. We will consider questions of global stability and persistence of a diffusive food chain. Our system is more general than that considered in [13] even when there is no diffusion. Basically, we extend the results of [13] to this more realistic diffusive food chain. Our approach involves an application of the invariance principle of reaction-diffusion equations [1], the construction of a Liapunov functional $[13,26]$, and applications of the dynamical system results established in [11] and [29].

The paper is organized as follows. In Section 2, we will present our model and state some preliminary results. Section 3 will be devoted to discussing the global stability of the unique positive homogeneous steady state of the system. Section 4 deals with persistence aspects of the system. The paper concludes with a brief discussion.

\section{Preliminaries}

In this paper we propose to study the global stability and persistence aspects of the following system of reaction-diffusion equations which may be viewed as a diffusive 
food chain model:

$$
\begin{array}{ll}
\dot{u}_{i}=d_{i} \Delta u_{i}+f_{i}(u), & (x, t) \in \Omega \times R_{+} \\
\partial u_{i} / \partial \nu=0, & \text { on } \partial \Omega \times R_{+} \\
u_{i}(x, 0)=u_{i 0}(x) \geq 0, & x \in \bar{\Omega}
\end{array}
$$

where $1 \leq i \leq n, 0 \leq d_{1} \leq d_{2} \leq \cdots \leq d_{n}$, and $u=\left(u_{1}, u_{2}, \ldots u_{n}\right)$. Here $\Omega$ is a bounded and connected domain in $R^{m}$ with smooth boundary, $\partial / \partial v$ denotes differentiation along the normal to $\partial \Omega$ and $\Delta$ is the Laplacian. The function $u_{i}(x, t)$ is the density distribution of the $i$ th population at time $t$. The boundary condition (2.2) implies that the system is closed (that is, no immigration and emigration). It is assumed that $u_{i 0}(x)$ is continuous. The functions $f_{i}(u), i=1, \ldots, n$, are assumed to take the form

$$
\begin{aligned}
& f_{1}(u)=u_{1} g_{1}\left(u_{1}\right)-\sum_{j=2}^{n} u_{j} p_{j 1}\left(u_{1}\right), \\
& f_{i}(u)=u_{i}\left[g_{i}\left(u_{i}\right)+\sum_{j=1}^{i-1} q_{i j}\left(u_{j}\right)\right]-\sum_{j=i+1}^{n} u_{j} p_{j i}\left(u_{i}\right), \quad i=2, \ldots, n-1, \\
& f_{n}(u)=u_{n}\left[g_{n}\left(u_{n}\right)+\sum_{j=1}^{n-1} q_{n j}\left(u_{j}\right)\right] .
\end{aligned}
$$

We will always assume that functions appearing in (2.4)-(2.6) are continuously differentiable with respect to their arguments. In order to ensure that (2.1)-(2.3) constitute a model for a food chain, we assume further that the following conditions are satisfied:

(A1) $g_{1}\left(u_{1}\right)$, the specific growth rate of the bottom prey, is assumed to satisfy $g_{1}:[0, \infty) \rightarrow R, g_{1}(0)>0, g_{1}^{\prime}\left(u_{1}\right)<0$; there exists $K>0$ such that $g_{1}(K)=0$.

(A2) $\quad p_{j i}\left(u_{i}\right)$ is the predator functional response of the $j$ th species on the $i$ th species. We assume $p_{j i}:[0, \infty) \rightarrow[0, \infty), p_{j i}(0)=0, p_{j i}^{\prime}\left(u_{i}\right)>0$.

(A3) $q_{i j}\left(u_{j}\right)$ is the conversion (or assimilation) function of the $i$ th species from the $j$ th species. Also, we assume $q_{i j}:[0, \infty) \rightarrow[0, \infty), q_{i j}(0)=0, q_{i j}^{\prime}\left(u_{j}\right)>0$.

(A4) $g_{i}\left(u_{i}\right), i \geq 2$, is the death rate function of the $i$ th species. It is assumed that $g_{i}(0)=-\delta_{i}<0, g_{i}^{\prime}\left(u_{i}\right) \leq 0$.

When $d_{i}=0, g_{i}\left(u_{i}\right)=-\delta_{i}, q_{i, i-1}\left(u_{i-1}\right)=c_{i} p_{i, i-1}\left(u_{i-1}\right), c_{i}>0, i \geq 2$, and $q_{i j}\left(u_{j}\right)=$ $p_{i j}\left(u_{j}\right)=0$ when $j \neq i-1$, system (2.1) reduces to the so-called simple food chain considered by Freedman and So in [13].

Clearly, our system (2.1)-(2.3) satisfies the so-called food pyramid condition (see [1]), that is, there exists a positive constant $b_{1}$ such that $u_{1}^{-1} f_{1}(u) \leq b_{1}$ for $u_{i} \geq 0$; 
also, given that $u_{1} \geq 0, \ldots, u_{i-1} \geq 0$ are in a bounded set $U_{i}$ in $R^{i-1}$, there exists a positive constant $b_{i}=b_{i}\left(U_{i}\right)$ such that

$$
u_{i}^{-1} f_{i}(u) \leq b_{i}, \quad i=2, \ldots, n .
$$

Thus, Theorem 2.1 in [1] implies that system (2.1)-(2.3) has a unique classical nonnegative solution $u(x, t)=\left(u_{1}(x, t), \ldots, n_{n}(x, t)\right)$ that exists for all time. Moreover, if $u_{i 0}(x) \neq \equiv$, then $u_{i}(x, t)>0$ for $t>0, x \in \bar{\Omega}$.

We call system (2.1) persistent if, for $u_{i 0}(x) \not \equiv 0,1 \leq i \leq n$,

$$
\inf _{x \in \bar{\Omega}} \liminf _{t \rightarrow+\infty} u_{i}(t, x)>0,
$$

and uniformly persistent, if for $u_{i 0}(x) \not \equiv 0,1 \leq i \leq n$,

$$
\liminf _{t \rightarrow+\infty}\left(\max _{x \in \hat{\Omega}} u_{i}(x, t)\right) \geq \sigma,
$$

where $\sigma$ is a positive constant independent of $u_{i 0}, x$ and $i$.

Intuitively, the persistence notion requires the existence of a positive constant, say $b$, such that at any location $x$, the lim inf of the solution is larger than $b$. In other words, eventually, the solution stays away from the boundary by a distance of at least $b$. The notion of uniform persistence, on the other hand, requires only that there is a $\sigma>0$, such that the maximum values of the solution over the domain at large times are no less than $\sigma$. This indicates that our persistence notion in fact implies that of uniform persistence. It is not clear at this moment whether uniform persistence implies persistence here.

It should be mentioned that the above definition for uniform persistence is consistent with that in Hale and Waltman [24]. However, our requirement for persistence is stronger than that in [24]. When $d_{i}=0$, these definitions coincide with those for strong persistence and uniform persistence, respectively.

LEMMA 2.1. In system (2.1)-(2.3), assume that (A1)-(A4) hold. Then, for any $\epsilon>0$, there is a $T_{0}=T_{0}\left(\epsilon, u_{10}\right)$, such that for $t \geq T_{0}, u_{1}(x, t)<K+\epsilon, x \in \bar{\Omega}$.

ProOF. Let $u(x, t)=\left(u_{1}(x, t), \ldots, u_{n}(x, t)\right)$ be the solution of (2.1)-(2.3). Denote

$$
u_{0}=\max _{x \in \tilde{\Omega}} u_{10}(x) .
$$

Let $\bar{u}_{1}(t)$ be the solution of the initial value problem (constant in $x$ )

$$
\begin{aligned}
& \dot{\bar{u}}_{1}=\bar{u}_{1} g_{1}\left(\bar{u}_{1}\right), \\
& \bar{u}_{1}(0)=u_{0} .
\end{aligned}
$$


Clearly, $\partial \bar{u}_{1}(t) / \partial v=0$ on $\partial \Omega \times R_{+}, \Delta \bar{u}_{1}(t)=0$, and for $(x, t) \in \Omega \times R_{+}$,

$$
\dot{\bar{u}}_{1}-d_{1} \Delta \bar{u}_{1}-f_{1}\left(\bar{u}_{1}, u_{2}(x, t), \ldots, u_{n}(x,(t)) \geq 0=\dot{u}_{1}-d_{1} \Delta u_{1}-f_{1}(u) .\right.
$$

Thus, by Theorem 10.1 in [46], we have

$$
u_{1}(x, t) \leq \bar{u}_{1}(t), \quad t \geq 0 .
$$

It is easy to see that, for any $\epsilon>0$, there is a $T_{0}=T_{0}\left(\epsilon, u_{10}\right)$ such that, for $t \geq T_{0}$,

$$
\bar{u}_{1}(t) \leq K+\epsilon .
$$

This, together with (2.13), completes the proof.

LEMMA 2.2. In system (2.1)-(2.3), assume that (A1)-(A4) hold, and (A5) there is a constant $\alpha>1$, such that

$$
\alpha p_{i j}>q_{i j}, \quad i>j, j=1, \ldots, n-1 .
$$

Denote $\delta=\min \left\{\left|g_{i}(0)\right|, \quad i=2, \ldots, n\right\}, M=\delta^{-1} \alpha^{n-1}(K+1)\left[\delta+g_{1}(0)\right] m(\Omega)$, where $m(\Omega)$ is the measure of $\Omega$, and

$$
Q(t)=\sum_{i=1}^{n} \int_{\Omega} \alpha^{n-i} u_{i}(x, t) d x .
$$

Then $\lim _{t \rightarrow+\infty} \sup Q(t) \leq M$.

PROOF. By a direct computation, we have

$$
\dot{Q}(t)=\sum_{i=1}^{n} \int_{\Omega} \alpha^{n-i} \dot{u}_{i}(x, t) d x=\sum_{i=1}^{n} \int_{\Omega} \alpha^{n-i} d_{i} \Delta u_{i} d x+\sum_{i=1}^{n} \int_{\Omega} \alpha^{n-i} f_{i}(u) d x .
$$

Since (2.2) must be satisfied, we have

$$
\int_{\Omega} \alpha^{n-i} d_{i} \Delta u_{i} d x=\alpha^{n-i} d_{i} \int_{\partial \Omega}\left(\partial u_{i} / \partial v\right) d s=0 .
$$

From Lemma 2.1, we see that there is a $T_{0}>0$ such that, for $t \geq T_{0}, u_{1}(x, t) \leq U_{1}=$ $K+1$. Thus, for $t \geq T_{0}$,

$$
\begin{aligned}
\sum_{i=1}^{n} \alpha^{n-i} f_{i}(u)= & \alpha^{n-1} u_{1} g_{1}\left(u_{1}\right)+\sum_{i=2}^{n} \alpha^{n-i} u_{i} g_{i}\left(u_{i}\right) \\
& +\sum_{i=2}^{n} \sum_{j=1}^{i-1}\left[\alpha^{n-i} q_{i j}\left(u_{j}\right)-\alpha^{n-j} p_{i j}\left(u_{j}\right)\right]
\end{aligned}
$$




$$
\begin{aligned}
& \leq \alpha^{n-1} u_{1} g_{1}\left(u_{1}\right)-\sum_{i=2}^{n} \delta \alpha^{n-i} u_{i} \\
& \leq-\delta \sum_{i=1}^{n} \alpha^{n-i} u_{i}+\alpha^{n-1} u_{1}\left[\delta+g_{1}\left(u_{1}\right)\right] \\
& \leq-\delta \sum_{i=1}^{n} \alpha^{n-i} u_{i}+\alpha^{n-1} U_{1}\left[\delta+g_{1}(0)\right]
\end{aligned}
$$

Therefore

$$
\dot{Q}(t) \leq-\delta Q(t)+\alpha^{n-1} U_{1}\left[\delta+g_{1}(0)\right] m(\Omega),
$$

where $m(\Omega)$ is the measure of $\Omega$. Let $G=\alpha^{n-1} U_{1}\left[\delta+g_{1}(0)\right] m(\Omega)$, then (2.19) implies

$$
Q(t) \leq e^{-\delta t} Q(0)+G \int_{0}^{t} e^{-\delta(t-s)} d s
$$

Therefore

$$
\lim _{t \rightarrow+\infty} \sup Q(t) \leq \delta^{-1} G \equiv M
$$

This proves the lemma.

If $d_{i}=0$ for some $i \geq 1$, then the above lemma alone indicates that $u_{j}(x, t)$, $j \leq i$, are bounded. In this case, the above lemma, together with Theorem 3.1 in [1], yields the boundedness of $u_{l}(x, t), l>i$, in the sense of $L_{\infty}$. Clearly, Theorem 2.1 in [1] implies $u_{l}(x, t), l>i$, are classical which in turn means that $u_{l}(x, t), l>i$, are bounded. If $d_{1}>0$, we see that the above lemma implies that solutions of system (2.1) $-(2.3)$ are $L_{1}$ bounded. Again, by [1, Theorem 3.1], we conclude that they are $L_{\infty}$ bounded. Since solutions of (2.1)-(2.3) are classical, this indeed means that all solutions of (2.1)-(2.3) are bounded. Thus, in both cases, solutions of (2.1)-(2.3) are bounded. By an argument similar to the proof of Lemma 2.1 in Hutson and Moran [29], we can show that solutions of (2.1)-(2.3) are bounded in $C^{1}\left(\bar{\Omega} \times R_{+}, R_{+}^{n}\right)$ for $t \geq 1$.

We can now state and prove our main theorem in this section.

THEOREM 2.1. In system (2.1)-(2.3), assume (A1)-(A5) hold. Then solutions exist for $t \geq 0$ and they are nonnegative, classical and bounded in $C^{1}\left(\bar{\Omega} \times R_{+}, R_{+}^{n}\right)$, for $t \geq 1$. Further, there exists a positive constant $U$, which is independent of initial value, such that

$$
\lim _{t \rightarrow+\infty} \sup \sum_{i=1}^{n} u_{i}(x, t)<U .
$$


PROOF. The first statement is just a summary of the previous discussion. In order to prove the second statement, we once more need Theorem 3.1 in [1], which says that for an equation of the form

$$
\dot{u}=d \Delta u+u B(x, t), \quad x \in \Omega \subset R^{m},
$$

with boundary and initial conditions of the form (2.2)-(2.3), if $B(x, t)<a, a$ is a constant, $B(x, t)$ is locally Lipschitz in $(x, t)$, and

$$
\sup _{t \geq 0} \int_{\Omega} u(x, t) d x<L,
$$

where $L$ is a positive constant. Then

$$
\sup _{t \geq 0}\|u(\cdot, t)\|_{L_{\infty}(\Omega)} \leq 2^{m+4} a L .
$$

Since our system (2.1)-(2.3) satisfies the food pyramid condition and for $t \geq T_{0}$, $u_{1}(x, t) \leq K+1$, we see that there exists $a_{1}>0$ such that, for $t \geq T_{0}, u_{2}(x, t)$ satisfies (2.21), where $B(x, t)$ is replaced by $f_{2}(u)$, and $f_{2}(u)<a_{1}$. By Lemma 2.2, we see there exists $L_{1}>0, T_{1} \geq T_{0}$, such that for $t \geq T_{1}$,

$$
\int_{\Omega} u_{2}(x, t) d x<L_{1}
$$

where $L_{1}$ is independent of initial value. Hence, Theorem 3.1 in [1] implies for $t \geq T_{1}$,

$$
\sup _{t \geq T_{1}}\left\|u_{2}(\cdot, t)\right\|_{L_{\infty}(\Omega)} \leq 2^{m+4} a_{1} L_{1} .
$$

Since $u(x, t)$ is classical, (2.23) is equivalent to

$$
u_{2}(x, t) \leq L_{2} \equiv 2^{m+4} a_{1} L_{1}, \quad t \geq T_{1} .
$$

By repeating this argument, we arrive at the conclusion that there exists a $U$, independent of initial value, such that

$$
\lim _{t \rightarrow \infty} \sup \sum_{i=1}^{n} u_{i}(x, t)<U .
$$

This completes the proof.

The above theorem implies that eventually all nonnegative solutions of (2.1)-(2.3) will assume their values in a compact set in $R_{+}^{n}$. This fact will be used in a discussion of uniform persistence in Section 4.

In the rest of this paper we will assume that (A1)-(A5) hold in system (2.1)-(2.3). 


\section{Global stability}

Clearly, system $(2.1)-(2.2)$ has $(0,0, \ldots, 0)$ and $(K, 0,0, \ldots 0)$ as two of its spatially homogeneous steady state solutions. It can certainly have many more such solutions. To obtain all these solutions will be difficult and tedious, if it is not impossible. For convenience, we assume that system (2.1)-(2.2) has a unique spatially homogeneous equilibrium $u^{*}=\left(u_{1}^{*}, u_{2}^{*}, \ldots, u_{n}^{*}\right)$ such that $u_{i}^{*}>0, i=1,2, \ldots n$. The objective of this section is to derive criteria for $u^{*}$ to be globally asymptotically stable with respect to initial condition (2.3), such that $u_{i 0}(x) \not \equiv 0, i=1, \ldots, n$. For this purpose, we adopt the approach of Freedman and So [13]. We consider first the so-called simple food chain as discussed by Freedman and So [13]. We assume for $i=1,2, \ldots, n$,

$$
q_{i j}\left(u_{j}\right)=p_{i j}\left(u_{j}\right)=0, \quad \text { if } j \neq i-1 ; g_{i}\left(u_{i}\right)=-\delta_{i}
$$

and denote for $i=1,2, \ldots, n-1$,

$$
p_{i}\left(u_{i}\right)=p_{i+1, i}\left(u_{i}\right) .
$$

Assume further, for $i=1,2, \ldots, n-1$,

$$
q_{i}\left(u_{i}\right)=q_{i+1, i}\left(u_{i}\right)=c_{i} p_{i}\left(u_{i}\right), \quad c_{i}>0 .
$$

Thus (2.1) reduces to

$$
\left\{\begin{array}{l}
\dot{u}_{1}=d_{1} \Delta u_{1}+u_{1} g_{1}\left(u_{1}\right)-u_{2} p_{1}\left(u_{1}\right) \\
\quad \vdots \\
\dot{u}_{i}=d_{i} \Delta u_{i}+u_{i}\left[-\delta_{i}+c_{i} p_{i-1}\left(u_{i-1}\right)\right]-u_{i+1} p_{i}\left(u_{i}\right) \\
\quad \vdots \\
\dot{u}_{n}=d_{n} \Delta u_{n}+u_{n}\left[-\delta_{n}+c_{n} p_{n-1}\left(u_{n-1}\right)\right] .
\end{array}\right.
$$

For $i=1, \ldots, n, i \neq n-1$, we define

$$
V_{i}\left(u_{i}\right)=u_{i}-u_{i}^{*} \ln u_{i} .
$$

It is easy to see $V_{i}\left(u_{i}\right)$ is bounded below by $u_{i}^{*}-u_{i}^{*} \ln u_{i}^{*}$ and tends to $+\infty$ as $u_{i} \rightarrow 0^{+}$ or $u_{i} \rightarrow+\infty$.

For $i=n-1$, we define (see Freedman and So [13])

$$
V_{n-1}\left(u_{n-1}\right)=\int_{u_{n-1}^{*}}^{u_{n-1}} \frac{-\delta_{n}+c_{n} p_{n-1}(\xi)}{p_{n-1}(\xi)} d \xi .
$$


From the assumed properties of $p_{n-1}\left(u_{n-1}\right)$, one can see that $V_{n-1}$ has properties similar to those of the other $V_{i}$ 's listed above.

Finally, we define on the space of $C\left(\bar{\Omega} \times R_{+}, R_{+}^{n}\right)$,

$$
W(u)(t)=\int_{\Omega} \sum_{i=1}^{n} V_{i}\left(u_{i}\right) d x .
$$

The derivative of $W(t)$ along any positive solution of (3.4) with (2.2)-(2.3) takes the form

$$
\dot{W}(u)(t)=\int_{\Omega} \sum_{i=1}^{n} V_{i}^{\prime}\left(u_{i}\right) d_{i} \Delta u_{i} d x+\int_{\Omega} \sum_{i=1}^{n} V_{i}^{\prime}\left(u_{i}\right) f_{i}(u) d x .
$$

By using the boundary condition (2.2), we have

$$
\int_{\Omega} \sum_{i=1}^{n} V_{i}^{\prime}\left(u_{i}\right) d_{i} \Delta u_{i} d x=-\sum_{i=1}^{n} \int_{\Omega} d_{i} V_{i}^{\prime \prime}\left(u_{i}\right)\left|\nabla u_{i}\right|^{2} d x,
$$

where

$$
\left|\nabla u_{i}\right|^{2}=\sum_{j=1}^{m}\left(\frac{\partial u_{i}}{\partial x_{j}}\right)^{2}
$$

For $i \neq n-1$, we have

$$
V_{i}^{\prime \prime}\left(u_{i}\right)=u_{i}^{*}\left(u_{i}^{-1}\right)^{2}>0,
$$

and for $i=n-1$, we have

$$
V_{n-1}^{\prime \prime}\left(u_{n-1}\right)=\delta_{n}\left(p_{n-1}\left(u_{n-1}\right)\right)^{-2} p_{n-1}^{\prime}\left(u_{n-1}\right)>0 .
$$

Thus, we have shown that

$$
\dot{W}(u)(t) \leq \int_{\Omega} \sum_{i=1}^{n} V_{i}^{\prime}\left(u_{i}\right) f_{i}(u) d x .
$$

As in Freedman and So [13, p. 76], we define the functions $\phi_{i}, \psi_{i}, i=1, \ldots, n-1$, as follows:

$$
\begin{aligned}
g_{1}\left(u_{1}\right)-u_{2}^{*} p_{1}\left(u_{1}\right) u_{1}^{-1} & =-\left(u_{1}-u_{1}^{*}\right) \psi_{1}\left(u_{1}\right), \\
-\delta_{i}+c_{i} p_{i-1}\left(u_{i-1}^{*}\right)-u_{i+1}^{*} p_{i}\left(u_{i}\right) u_{i}^{-1} & =-\left(u_{i}-u_{i}^{*}\right) \psi_{i}\left(u_{i}\right), \quad i=2, \ldots, n-2 ; \\
\frac{u_{n-1}}{p_{n-1}\left(u_{n-1}\right)}\left[-\delta_{n-1}+c_{n-1} p_{n-2}\left(u_{n-2}^{*}\right)\right]-u_{n}^{*} & =-\left(u_{n-1}-u_{n-1}^{*}\right) \psi_{n-1}\left(u_{n-1}\right),
\end{aligned}
$$




$$
\begin{aligned}
p_{i}\left(u_{i}\right)-p_{i}\left(u_{i}^{*}\right) & =\left(u_{i}-u_{i}^{*}\right) \phi_{i}\left(u_{i}\right), \quad i=1, \ldots, n-2, \\
-\delta_{n}+c_{n} p_{n-1}\left(u_{n-1}\right) & =\left(u_{n-1}-u_{n-1}^{*}\right) \phi_{n-1}\left(u_{n-1}\right) .
\end{aligned}
$$

We denote

$$
v_{i}=u_{i}-u_{i}^{*}, \quad i=1, \ldots, n
$$

By the same algebraic manipulations as documented in [13, p. 74-76], we can write

$$
\sum_{i=1}^{n} V_{i}^{\prime}\left(u_{i}\right) f_{i}(u)=-\sum_{i, j=1}^{n-1} a_{i j}(u) v_{i} v_{j}
$$

where $a_{i j}(u)=a_{j i}(u), a_{i j}=0$ if $|i-j|>1$, and

$$
\begin{gathered}
a_{i i}(u)=\psi_{i}\left(u_{i}\right), i=1, \ldots, n-2, \quad a_{n-1, n-1}(u)=\phi_{n-1}\left(u_{n-1}\right) \psi_{n-1}\left(u_{n-1}\right), \\
a_{i, i+1}(u)=\frac{1}{2}\left[p_{i}\left(u_{i}\right) u_{i}^{-1}-c_{i+1} \phi_{i}\left(u_{i}\right)\right], \quad i=1, \ldots, n-3, \\
a_{n-2, n-1}(u)=\frac{1}{2}\left[\frac{p_{n-2}\left(u_{n-2}\right)}{u_{n-2}}-\frac{c_{n-1} u_{n-1}}{p_{n-1}\left(u_{n-1}\right)} \phi_{n-2}\left(u_{n-2}\right) \phi_{n-1}\left(u_{n-1}\right)\right] .
\end{gathered}
$$

Finally, we denote $A$ to be the $(n-1) \times(n-1)$ matrix $A=\left(a_{i j}\right)$.

Now we are ready to state and prove the main result of this section, which reduces to Theorem 3.1 in [13] when $d_{i}=0, i=1, \ldots, n$.

THEOREM 3.1. Consider system (3.4) with boundary condition (2.2). Assume (A1)(A4) hold and $A$ is positive definite for all $u \in \operatorname{Int} R_{+}^{n}$. Then $u^{*}$ is uniformly globally asymptotically stable (in the norm of $C\left(\bar{\Omega} \times R_{+}, R_{+}^{n}\right)$ ) with respect to a nonnegative initial function $u_{0}(x)$, such that $u_{i 0}(x) \not \equiv 0, i=1, \ldots, n, x \in \bar{\Omega}$.

PROOF. We define a Liapunov functional $W(u)(t)$ as in (3.7). Thus, from (3.12), we have

$$
\dot{W}(u)(t) \leq-\int_{\Omega} \sum_{i, j=1}^{n-1} a_{i j}(u) v_{i} v_{j} d x,
$$

where $v_{i}$ is defined as in (3.13). Since $A$ is assumed to be definite, we see that if $\dot{W}(u)(t)=0$, then (since $u$ is a classical solution)

$$
v_{1}=v_{2}=\cdots=v_{n-1}=0,
$$

that is,

$$
u_{1}=u_{1}^{*}, \ldots, u_{n-1}=u_{n-1}^{*} .
$$


Thus, in the space of $C\left(\bar{\Omega} \times R_{+}, R_{+}^{n}\right)$, we have

$$
E=\{u: \dot{W}(u)(t)=0\} \subset S=\left\{u: u_{1}=\cdots=u_{n-1}=0, \nabla u_{n}=0\right\} .
$$

Let $I$ be the largest invariant set (with respect to (3.4)) in $E$. We see that $u \in I$ implies

$$
u=\left(u_{1}^{*}, \ldots, u_{n-1}^{*}, \bar{u}_{n}\right), \quad \bar{u}_{n} \text { is a constant. }
$$

Thus

$$
0=\ddot{u}_{n-1}^{*}=d_{n-1} \Delta u_{n-1}^{*}+u_{n-1}^{*}\left[-\delta_{n-1}+c_{n-1} p_{n-2}\left(u_{n-2}^{*}\right)\right]-\bar{u}_{n} p_{n-1}\left(u_{n-1}^{*}\right),
$$

which implies

$$
\bar{u}_{n}=u_{n}^{*} \text {. }
$$

By the invariance principle, Theorem 4.3.4 in Henry [27], we conclude that for an initial function $u_{0}(x) \geq 0$, such that $u_{i 0}(x) \not \equiv 0, i=1, \ldots, n, x \in \Omega$, the solution $u(x, t)$ of $(3.4),(2.2)-(2.3)$ satisfies

$$
u(x, t) \rightarrow u^{*} \text { as } t \rightarrow+\infty \text { in } L_{1} \text { norm. }
$$

By Theorem 2.1 in the previous section, we see that (3.23) is equivalent to

$$
\lim _{t \rightarrow+\infty} u(x, t)=u^{*} \text { uniformly on } \bar{\Omega} \text {. }
$$

This completes the proof.

It should be mentioned here that the remarks and corollary following Theorem 3.1 in [13] are equally applicable to the above theorem. They are very useful in the application of the result. We omit them here just to avoid repetition.

It is easy to see that, in general, one can try to use

$$
W(u)(t)=\int_{\Omega} \sum_{i=1}^{n} c_{i} V_{i}\left(u_{i}\right) d x
$$

as a trial Liapunov functional, where $c_{i}, i=1, \ldots, n$, are positive constants. This may give better results than Theorem 3.1.

The next theorem deals with the general model (2.1)-(2.3). Note that the proof uses a slightly different Liapunov functional and hence it is independent of Theorem 3.1. The result may be too general to be applicable.

THEOREM 3.2. In (2.1)-(2.3), assume there exist positive constants $c_{i}, i=1, \ldots, n$, such that

$$
\sum_{i=1}^{n} c_{i}\left(u_{i}-u_{i}^{*}\right) u_{i}^{-1} f_{i}(u) \leq 0
$$


and the largest invariant set in

$$
E=\left\{u: \sum_{i=1}^{n} c_{i}\left(u_{i}-u_{i}^{*}\right) u_{i}^{-1} f_{i}(u)=0\right\}
$$

is $u^{*}$. Then $E^{*}$ is uniformly globally asymptotically stable (in the norm of $C(\bar{\Omega} \times$ $\left.R_{+}, R_{+}^{n}\right)$ ) with respect to a nonnegative initial function $u_{0}(x)$, such that $u_{i 0}(x) \not \equiv 0$, $i=1, \ldots, n, x \in \bar{\Omega}$.

PROOF. We define a Liapunov functional $W(u)(t)$ for $(2.1)-(2.2)$ as

$$
W(u)(t)=\int_{\Omega} \sum_{i=1}^{n} c_{i}\left(u_{i}-u_{i}^{*} \ln u_{i}\right) d x .
$$

From (3.26), we have

$$
\dot{W}(u)(t) \leq \int_{\Omega} \sum_{i=1}^{n} c_{i}\left(u_{i}-u_{i}^{*}\right) u_{i}^{-1} f_{i}(u) \leq 0 .
$$

The rest of the proof is the same as that for Theorem 3.1. We omit it here.

In the following, we consider the frequently used diffusive Lotka-Volterra food chain, that is, $u_{i}^{-1} f_{i}(u)$ are linear functions of $u_{i}$. We may assume

$$
\begin{cases}g_{1}\left(u_{1}\right)=g_{1}\left(1-u_{1} / K\right), \quad g_{i}\left(u_{i}\right)=-\delta_{i}-g_{i} u_{i}, & i=2, \ldots, n, \\ p_{j i}\left(u_{i}\right)=p_{j i} u_{i}, \quad q_{j i}\left(u_{i}\right)=q_{j i} u_{i}, & i<j,\end{cases}
$$

where $g_{1}>0, g_{i} \geq 0, i=2, \ldots, n ; p_{j i}>0, q_{j i}>0, i \leq j$ are all constants.

For any positive constants $c_{i}>0, i=1, \ldots, n$, we have

$$
\begin{aligned}
\sum_{i=1}^{n} c_{i}\left(u_{i}-u_{i}^{*}\right) u_{i}^{-1} f_{i}(u) & \\
= & c_{1}\left(u_{1}-u_{1}^{*}\right)\left[-g_{1}\left(u_{1}-u_{1}^{*}\right) / K-\sum_{j=2}^{n} p_{j 1}\left(u_{j}-u_{j}^{*}\right)\right] \\
& +\sum_{i=2}^{n-1} c_{i}\left(u_{i}-u_{i}^{*}\right)\left[-g_{i}\left(u_{i}-u_{i}^{*}\right)+\sum_{j=1}^{i-1} g_{i j}\left(u_{j}-u_{j}^{*}\right)-\sum_{j=i+1}^{n} p_{j i}\left(u_{j}-u_{j}^{*}\right)\right] \\
& +c_{n}\left(u_{n}-u_{n}^{*}\right)\left[-g_{n}\left(u_{n}-u_{n}^{*}\right)+\sum_{j=1}^{n-1} q_{n j}\left(u_{j}-u_{j}^{*}\right)\right] .
\end{aligned}
$$

Let $v_{i}$ be defined as in (3.13), then we can rewrite the above equation as

$$
\sum_{i=1}^{n} c_{i}\left(u_{i}-u_{i}^{*}\right) u_{i}^{-1} f_{i}(u)=\frac{1}{2} v\left(A^{T} C+C A\right) v^{T},
$$


where $v=\left(v_{1}, \ldots, v_{n}\right), C=\operatorname{diag}\left[c_{1}, c_{2}, \ldots, c_{n}\right]$ and $A=\left(a_{i j}\right)_{n \times n}$ is defined as

(i) $a_{11}=-g_{1} / K, a_{i i}=-g_{i}$;

(ii) $a_{i j}= \begin{cases}q_{i j}, & j=1, \ldots, i-1, \\ -p_{j i}, & j=i+1, \ldots, n .\end{cases}$

Thus we have the following result.

THEOREM 3.3. Assume (3.29) holds in system (2.1)-(2.3). If there exists a positive diagonal matrix $C$ such that $A^{T} C+C A$ is negative definite, then the conclusion of Theorem 3.2 holds.

PROOF. This is an immediate corollary of Theorem 3.2.

As an example to the above theorem, consider the simple three level food chain where

$$
\begin{aligned}
& f_{1}(u)=u_{1}\left(5-2 u_{1}-2 u_{2}\right), \\
& f_{2}(u)=u_{2}\left(-2-u_{2}+4 u_{1}-u_{3}\right), \\
& f_{3}(u)=u_{3}\left(-1-u_{3}+2 u_{3}\right) .
\end{aligned}
$$

Then we have $a_{11}=-2, a_{22}=a_{33}=-1, a_{12}=-2, a_{13}=0, a_{21}=4, a_{23}=-1$, $a_{31}=0, a_{32}=2$. This food chain has a homogeneous positive steady state $(2,1 / 2,6)$. For this particular food chain, we can chose $C=\operatorname{diag}[1,1 / 2,1 / 4]$. Then it is easy to see that $A^{r} C+C A=\operatorname{diag}[-4,-1,-1 / 2]$. Theorem 3.3 implies that the homogeneous positive steady state is globally asymptotically stable.

REMARK 3.1. It can be seen from our definition of $W(u)(t)$ in (3.27) that we can in fact allow $u_{i}^{*}=0$, for some $i \in\{1,2, \ldots, n\}$, in our discussions.

\section{Persistence}

For simplicity, in this section, we will restrict our attention to the diffusive simple food chain; that is, system (3.4) with boundary and initial conditions (2.2)-(2.3). As will be seen in the following discussion, a detailed analysis of the persistence aspect of the full system (2.1)-(2.3) will be rather complicated, if not impossible. Our objective is to derive criteria for system (3.4) to be persistent or uniformly persistent. Our persistence result is a direct extension of the work of Freedman and So [13], while the uniform persistence result is a direct application of a criterion established in Hutson and Moran [29].

In order to state and prove our persistence result, we need the following notation and a lemma from Dunbar et al. [11]. 
Let $X$ be a metric space and $D$ an open subset of $R^{+} \times X$. A mapping $\pi: D \rightarrow X$ is called a local semiflow on $X$ if the following properties hold:

(i) $\pi$ is continuous;

(ii) for every $x \in X$, there exists $\omega_{x}, 0<\omega_{x} \leq \infty$, such that $(t, x) \in D$ if and only if $0 \leq t<\omega_{x}$

(iii) $\pi(0, x)=x$, for all $x \in X$;

(iv) if $(t, x) \in D$ and $(s, \pi(t, x)) \in D$, then $(t+s, x) \in D$ and $\pi(t+s, x)=$ $\pi(s, \pi(t, x))$.

For convenience, in the following we use the convention $x \pi t:=\pi(t, x)$. Let $J$ be a real interval and let $\sigma: J \rightarrow X$ be a mapping. We call $\sigma$ a solution of $\pi$ if, for all $t \in J, s \in[0, \infty)$ for which $t+s \in J$, it follows that $\sigma(t) \pi s$ is defined and $\sigma(t) \pi s=\sigma(t+s)$. If $J=R$, then $\sigma$ is called a full solution. If $Y \subset X$, we define

$$
\begin{gathered}
A^{+}(Y)=\left\{x \in X: x \pi\left[0, \omega_{x}\right) \subset Y\right\}, \\
A^{-}(Y)=\left\{x \in X: \text { there is a solution } \sigma=R^{-} \rightarrow X \text { with } \sigma(0)=x,\right. \\
\text { and } \left.\sigma\left(R^{-}\right) \subset Y, \text { where } R^{-}=(-\infty, 0]\right\}, \\
A(Y)=A^{+}(Y) \cap A^{-}(Y) .
\end{gathered}
$$

We call $Y$ positively invariant (with respect to $\pi$ ) if $A^{+}(Y)=Y$, negatively invariant if $A^{-}(Y)=Y$, and invariant if $A(Y)=Y$. It is important to note that if $\omega_{x}=+\infty$ for all $x \in Y$, then $Y$ is invariant if and only if for each $x \in Y$, there exists a full solution $\sigma$ with $\sigma(0)=x$ and $\sigma(R) \subset Y$.

We say that the local semiflow $\pi$ does not explode in $Y$, if for every $x \in x \pi\left[0, \omega_{x}\right) \subset Y$ we have that $\omega_{x}=\infty$.

If $\sigma$ is a solution on $R^{+}$(respectively $R^{-}$), then we denote its $\omega$-limit set ( $\alpha$-limit set) by $\omega(\sigma)(\alpha(\sigma))$.

Next, let $K$ be a closed invariant set, then $K$ is called an isolated invariant set if there exists a closed set $N$ such that $K \subset \operatorname{Int} N$ and $K=A(N)$. A closed set $N$ is called an isolating neighborhood if $K=A(N) \subset \operatorname{Int} N$. Clearly, a hyperbolic equilibrium point of $\pi$ is an isolated invariant set.

The following result on semiflows is established in [11, Theorem 2.2].

LEMMA 4.1. Let $K$ be an isolated invariant set and let $N$ be an isolating neighborhood of $K$. Suppose that $\pi$ does not explode in $N$. Let $y \in X$ be a point for which $\omega_{y}=\infty$ and $y \pi\left(R^{+}\right)$is precompact. Let $\sigma(t)=y \pi t, t \geq 0$, and suppose that

$$
\omega(\sigma) \cap K \neq \phi, \quad \omega(\sigma) \cap(X \backslash K) \neq \phi .
$$

Then

$$
\omega(\sigma) \cap A^{+}(N) \cap \partial N \neq \phi \quad \text { and } \quad \omega(\sigma) \cap A^{-}(N) \cap \partial N \neq \phi .
$$


$A^{+}(N) \backslash K$ and $A^{-}(N) \backslash K$ are called the stable and unstable manifold of $K$ (relative to $N)$. Intuitively, Lemma 4.1 says that $\omega(\sigma)$ contains points from both the stable and unstable manifolds of $K$, whenever it contains points of $K$ and its complement.

Finally, for $\left\{i_{1}, \ldots, i_{k}\right\} \subset\{1, \ldots, n\}$, we define a subspace of $C\left(\bar{\Omega}, R_{+}^{n}\right)$,

$$
H_{i_{1}, \ldots, i_{k}}=\left\{u: u_{i}=0 \text { for all } i \notin\left\{i_{1}, \ldots, i_{k}\right\}\right\} .
$$

For a simple food chain (3.4), it is easy to see that its spatial homogeneous steady states (if they exist) must take the form $E_{k}\left(u_{1}^{(k)}, \ldots, u_{k}^{(k)}, 0, \ldots, 0\right), u_{i}^{(k)}>0,1 \leq i \leq k$, and $1 \leq k \leq n$, or $(0, \ldots, 0) \equiv E_{0}$. We say $E_{i}$ is hyperbolic if it, as the equilibrium of $\dot{u}_{i}=f_{i}(u)$, is hyperbolic. We will use the same notation $E_{k}$ to denote the function $u(x) \in C\left(\bar{\Omega}, R_{+}^{n}\right)$, where $u_{i}(x) \equiv u_{i}^{(k)}$. This should not cause any confusion.

THEOREM 4.1. For system (3.4), (2.2)-(2.3), assume that $E_{i}, i=1, \ldots, n-1$, exist and are hyperbolic. Further, suppose that $E_{i}$ are globally asymptotically stable with respect to Int $H_{1, \ldots, i}$. Then if

$$
-\delta_{n}+c_{n} p_{n-1}\left(u_{n-1}^{(n-1)}\right)>0,
$$

the system (3.4), (2.2)-(2.3) is persistent with respect to $C\left(\bar{\Omega}, R_{+}^{n}\right)$.

ProOF. Suppose that system (3.4) is not persistent. Then there is a $\tilde{u}_{0}(x) \epsilon$ $C\left(\bar{\Omega}, R_{+}^{n}\right), \tilde{u}_{i 0}(x) \not \equiv 0, i=1,2, \ldots, n$, such that for some $j \in\{1, \ldots, n\}$, there are sequences $t_{n} \rightarrow \infty, x_{n} \in \bar{\Omega}$ with $\tilde{u}_{j}\left(x_{n}, t_{n}\right) \rightarrow 0$ as $n \rightarrow \infty$, where $\tilde{u}(x, t)=\tilde{u}_{0}(x) \pi t$. By our Theorem 2.1, we know $\tilde{u}(x, t)$ is precompact in $C\left(\bar{\Omega}, R_{+}^{n}\right)$, thus we may assume that $\tilde{u}\left(\cdot, t_{n}\right) \rightarrow \tilde{w}^{0} \in \omega\left(\tilde{u}_{0}\right)$ and $x_{n} \rightarrow x_{0} \in \bar{\Omega}$ as $n \rightarrow+\infty$. Then $\tilde{w}_{j}^{0}\left(x_{0}\right)=0$, which by the invariance of the $\omega$-limit set, implies that $\tilde{w}_{j}^{0}(x) \equiv 0$. That is, $\tilde{w}^{0}(x)$ lies in $R_{+}^{n-1}$.

Clearly, there exist $i_{1}<i_{2}<\ldots<i_{k},\left\{i_{1}, \ldots, i_{k}\right\} \subset\{1,2, \ldots, n\}$, such that $\tilde{w}^{0}(\cdot) \in H_{i_{1}, \ldots, i_{k}}$.

We observe that if $u \in H_{1, \ldots, i-1, i+1, \ldots, k}$, then $\omega(u) \subset H_{1, \ldots, \lambda-1}$. And if $u \in H_{2, \ldots k}$, then $\omega(u)=E_{0}$. This can be shown by using the comparison principle, [46, Theorem 10.1]. Thus, indeed, there exists a unique $l<n$, such that $\omega\left(\tilde{w}^{0}\right) \subset H_{1, \ldots, l}$, and $\omega\left(\tilde{w}^{0}\right) \cap$ Int $H_{1 \ldots . l l} \neq \phi$, or $\omega\left(\tilde{w}^{0}\right)=E_{0}$. In both cases, the above argument indicates that there are $E_{i}, i=0, \ldots, n-1$, such that $E_{i} \in \omega\left(\tilde{w}^{0}\right) \subset \omega\left(\tilde{u}_{0}\right)$. For convenience, in the following we denote $W^{+}\left(E_{i}\right)$ and $W^{-}\left(E_{i}\right)$ as the stable and unstable manifolds of $E_{i}$ in $C\left(\bar{\Omega}, R_{+}^{n}\right)$, respectively.

Assume $E_{0} \in \omega\left(\tilde{u}_{0}\right)$. It is easy to see that the unstable manifold of $E_{0}$ is $H_{1}$ and its stable manifold is $H_{2,3, \ldots, n}$. By Lemma 4.1, there exists a $v_{1}$, such that

$$
v_{1} \in \omega\left(\tilde{u}_{0}\right) \cap H_{2,3 \ldots, n} \backslash\left\{E_{0}\right\} .
$$


Since $v_{1} \pi R \subset \omega\left(\tilde{u}_{0}\right)$, this implies $v_{1} \pi R$ is bounded. Thus the invariance of $\omega\left(\tilde{u}_{0}\right)$ implies that $\alpha\left(v_{1}\right)$ is nonempty compact and invariant, and $E_{0} \notin \alpha\left(v_{1}\right)$. However, this means that for $v_{0} \in \alpha\left(v_{1}\right), \omega\left(v_{0}\right) \subset \alpha\left(v_{1}\right)$, which leads to $E_{0} \notin \omega\left(v_{0}\right)$. This clearly contradicts our observation that if $v_{1} \in H_{2,3, \ldots, n}$, then $\omega\left(v_{1}\right)=E_{0}$. Therefore we have shown that $E_{0} \notin \omega\left(\tilde{u}_{0}\right)$.

Suppose now that $E_{1} \in \omega\left(\tilde{u}_{0}\right)$. By the hyperbolicity of $E_{1}$ and Lemma 4.1, there exists $Q_{1}^{+} \in W^{+}\left(E_{1}\right) \backslash\left\{E_{1}\right\}$ such that $Q_{1}^{+} \in \omega\left(\tilde{u}_{0}\right)$. By the hyperbolicity of $E_{1}$ and the global stability of $E_{2}$ in $H_{1,2}$, we see that $-\delta_{2}+c_{2} p_{1}\left(u_{1}^{(1)}\right)>0$. Therefore $W^{+}\left(E_{1}\right)=H_{1,3 \ldots, n} \backslash H_{3, \ldots, n}$ from our previous observation. If $Q_{1}^{+} \notin H_{1}$, then we can show that the full solution through $Q_{1}^{+}$must be unbounded by considering $\alpha\left(Q_{1}^{+}\right)$ as above. This is because the $\omega$-limit set of any solution passing through a point in $\alpha\left(Q_{1}^{+}\right)$must remain in $\alpha\left(Q_{1}^{+}\right)$, which does not contain $E_{1}$. This is impossible, since $H_{1,3, \ldots, n}$ is invariant, and $\alpha\left(Q_{1}^{+}\right)$will remain in it, which implies that for any $v_{2} \in \alpha\left(Q_{1}^{+}\right), \omega\left(v_{2}\right) \subset H_{1}$, and since $E_{1} \notin \omega\left(v_{2}\right)$, this leads to $\omega\left(v_{2}\right)=E_{0}$; therefore, $E_{0} \in \omega\left(v_{2}\right) \subset \alpha\left(Q_{1}^{+}\right) \subset \omega\left(\tilde{u}_{0}\right)$, a contradiction. Now, if $Q_{1}^{+} \in H_{1}$, then it is easy to see that closure of any orbit in $H_{1} \backslash\left\{E_{1}\right\}$ is either unbounded or contains $E_{0}$, which in both cases leads to a desired contradiction. Hence, $E_{1} \notin \omega\left(\tilde{u}_{0}\right)$.

Similarly, we can show that $E_{2}, \ldots, E_{n-2} \notin \omega\left(\tilde{u}_{0}\right)$. Further, because of the assumption that $E_{i}$ are globally asymptotically stable in $H_{1, \ldots, i}, i=1, \ldots, n-2$, no point of $H_{1, \ldots, i}$ can be in $\omega\left(\tilde{u}_{0}\right)$. Therefore no point of $H_{1, \ldots, i-1, i+1, \ldots, n}$ can be in $\omega\left(\tilde{u}_{0}\right)$.

Finally, if $E_{n-1} \in \omega\left(\tilde{u}_{0}\right)$, then by (4.2) and the global stability of $E_{n-1}$ in Int $H_{1, \ldots, n-1}$, we claim that $W^{+}\left(E_{n-1}\right)=$ Int $H_{1, \ldots, n-1} \backslash\left\{E_{n-1}\right\}$. Otherwise, arbitrarily close to $E_{n-1}$, there is a solution $u(x, t), \lim _{t \rightarrow+\infty} u(x, t)=E_{n-1}$ uniformly on $\bar{\Omega}$ and $u_{n}(x, t)>0$ for $t>0$. Clearly, there is an $\epsilon>0, T>0$, such that for $t \geq T, x \in \bar{\Omega}$,

$$
-\delta_{n}+c_{n} p_{n-1}\left(u_{n-1}(x, t)\right)>\epsilon
$$

and

$$
\sum_{i=1}^{n} u_{i}(x, t) \leq \epsilon
$$

Denote

$$
\mu=\min _{x \in \bar{\Omega}} u_{n}(x, T)>0 .
$$

Let $\sigma(t)$ be the solution of

$$
\dot{\sigma}(t)=\epsilon \sigma(t), \quad \sigma(T)=\mu .
$$

Denoting $\sigma(x, t) \equiv \sigma(t)$, we have

$$
\begin{gathered}
\dot{\sigma}-d_{n} \Delta \sigma-\epsilon \sigma=0, \\
\dot{u}_{n}-d_{n} \Delta u_{n}-\epsilon u_{n} \geq \dot{u}_{n}-d_{n} \Delta u_{n}-u_{n}\left[-\delta_{n}+c_{n} p_{n-1}\left(u_{n-1}\right)\right]=0 .
\end{gathered}
$$


Then the comparison result [46, Theorem 10.1] implies that

$$
u_{n}(x, t) \geq \sigma(t)=\mu e^{\epsilon(t-T)}, \quad x \in \bar{\Omega}, \quad t \geq T .
$$

Therefore

$$
\lim _{t \rightarrow+\infty} u_{n}(x, t)=+\infty
$$

a contradiction to (4.4).

Now, since $W^{+}\left(E_{n-1}\right)=$ Int $H_{1, \ldots, n-1} \backslash\left\{E_{n-1}\right\}$, by Lemma 4.1, there exist $Q_{n-1}^{+} \in$ $W^{+}\left(E_{n-1}\right)$, such that $Q_{n-1}^{+} \in \omega\left(\tilde{u}_{0}\right)$. But the closed orbit through $Q_{n-1}^{+}$either is unbounded or contains points of $H_{1, \ldots, i-1, i+1, \ldots, n-1}$ for some $i \leq n-2$. This again leads to a contradiction, proving the theorem.

In the rest of this section we assume that in the growth process of every species $u_{i}$ of system (3.4), a self crowding effect takes place, and

$$
\lim _{u_{i} \rightarrow+\infty} g_{i}\left(u_{i}\right)=-\infty
$$

We hereafter replace $-\delta_{i}$ by $g_{i}\left(u_{i}\right)$ in (3.4) and denote the resulting system by $\left(3.4^{\prime}\right)$. We assume further that

(H1) $\Omega$ is a connected and bounded open domain in $R^{m}$ with $C^{2}$ boundary. For each $i \in\{1, \ldots, n\}, f_{i} \in C^{2}\left(R_{+}^{n}, R\right), d_{i}>0, i=1, \ldots, n$.

LEMMA 4.2. Assume (4.7) holds in system (3.4'), then it has an arbitrary large invariant set of the form

$$
\Sigma=\bigcap_{i=1}^{n}\left\{u: 0 \leq u_{i} \leq l_{i}\right\} .
$$

$B y$ an invariant set, we mean that $f(u)$ points strictly into $\Sigma$ on $\partial \Sigma$.

PROOF. Since $\lim _{u_{1} \rightarrow \infty} g_{1}\left(u_{1}\right)=-\infty$, we can choose a positive constant $l_{1}$, such that $g_{1}\left(l_{1}\right)<0$. Clearly $f(u)$ points strictly into the space between $u_{1}=0$ and $u_{1}=l_{1}$ in $R_{+}^{n}$ from the side of $u_{1}=l_{1}$.

Since $\lim _{u_{2} \rightarrow \infty} g_{2}\left(u_{2}\right)=-\infty$, we can choose a positive constant $l_{2}$, such that

$$
g_{2}\left(l_{2}\right)+c_{2} p_{1}\left(l_{1}\right)<0 .
$$

Intuitively, this ensures that $f(u)$ points strictly into the space between $u_{2}=0$ and $u_{2}=l_{2}$ in $R_{+}^{n}$ from the side of $u_{2}=l_{2}$.

By repeating the above process, we can choose $l_{i}, i \geq 3$, inductively, so that

$$
g_{i}\left(l_{i}\right)+c_{i} p_{i-1}\left(l_{i-1}\right)<0 .
$$


One can see that

$$
\Sigma=\bigcap_{i=1}^{n}\left\{u: 0 \leq u_{i} \leq l_{i}\right\}
$$

is the desired invariant set. Clearly, it can be made as large as one wants.

It is easy to see from the above argument that Lemma 4.2 is valid for system (2.1), provided that (4.7) holds.

In order to state and prove our next result we need the following notation from Hutson and Moran [29].

For $Y \subset R_{+}^{n}$, define

$$
\begin{gathered}
X_{0}=\left\{u \in C\left(\bar{\Omega}, R^{n}\right): u(x) \in Y, \text { for } x \in \bar{\Omega}\right\}, \\
S_{0}=\left\{u \in X_{0}: \text { for some } i, u_{i}(x) \equiv 0, \text { for } x \in \bar{\Omega}\right\}, \\
X=X_{0} \pi[1, \infty), \quad S=S_{0} \pi[1, \infty), \\
\omega(V)=U_{v \in V} \omega(v), \text { where } V \subset C\left(\bar{\Omega}, R^{n}\right) .
\end{gathered}
$$

The following result is essentially contained in the proof of Lemma 5.1 in [29]. For completeness, we will sketch the proof.

LEMMA 4.3. Assume (H1) holds in system (2.1)-(2.3) and the system has an invariant set $X_{0}$ attracting all nonnegative solutions. Suppose that $\omega(\bar{S})$ consists of a finite number of spatially homogeneous steady states, $\bar{u}_{1}, \ldots, \bar{u}_{k}$, say. Then system (2.1) is uniformly persistent if and only if there exist $\alpha_{1}, \ldots, \alpha_{n}>0$, such that

$$
\sum_{i=1}^{i=n} \alpha_{i} F_{i}\left(\bar{u}_{j}\right)>0, \quad j=1, \ldots, k,
$$

where $F_{i}(u)=u_{i}^{-1} f_{i}(u)$.

PROOF. Following the proof of Lemma 5.1 in [29], we see all conditions of Theorem 3.2 in [29] are satisfied. Thus there is a compact set $M$ absorbing for $\bar{X} \backslash \bar{S}$ with

$$
d(M, \bar{S})=\min _{u \in M} d(u, \bar{s}) \equiv \sigma>0 .
$$

This clearly implies that

$$
\liminf _{t \rightarrow+\infty}\left(\max _{x \in \tilde{\Omega}} u_{i}(x, t)\right) \geq \sigma
$$

proving the lemma. 
Now we are ready to state and prove our uniform persistence result.

THEOREM 4.2. Assume (4.7) and (H1) hold in system (3.4'). Assume further that $E_{i}, i=1, \ldots, n-1$ (as in Theorem 4.1), exist and are hyperbolic and that $E_{i}$ are globally asymptotically stable with respect to Int $H_{1, \ldots, i}$. Then if

$$
g_{n}(0)+c_{n} p_{n-1}\left(u_{n-1}^{(n-1)}\right)>0,
$$

the system (3.4'), (2.2)-(2.3) is uniformly persistent.

PROOF. Clearly, all conditions of Lemma 4.3, except (4.9), are satisfied by (3.4').

We choose $\alpha_{1}=1$. It is easy to see that there exists an $\epsilon_{2}$ satisfying $0<\epsilon_{2}<1$, such that if $\alpha_{i} \leq \epsilon_{2}, i=2, \ldots, n$, then (4.9) is true for $E_{0}=(0, \ldots, 0)$. We may thus choose $\alpha_{2}=\epsilon_{2}$.

We observe that for $j \geq 1$,

$$
F_{1}\left(E_{j}\right)=\left(u_{1}^{(j)}\right)^{-1} f_{1}\left(E_{j}\right) \equiv 0,
$$

and $F_{2}\left(E_{1}\right)>0$, since $E_{2}$ exists and is globally stable in Int $H_{1,2}$. This implies there exists an $\epsilon_{3}$ satisfying $0<\epsilon_{3}<\epsilon_{2}$, such that if $\alpha_{i} \leq \epsilon_{3}, i=3, \ldots, n$, then (4.9) holds for $E_{1}$. Then we define $\alpha_{3}=\epsilon_{3}$.

Repeating the above argument, we can define $\alpha_{i}, i=1, \ldots, n-1$, such that

$$
1=\alpha_{1}>\alpha_{2}>\cdots>\alpha_{n-1}
$$

and (4.9) holds for $E_{0}, \ldots, E_{n-3}$, provided $\alpha_{n} \leq \alpha_{n-1}$. Since $F_{n-1}\left(u_{n-1}^{(n-2)}\right)>0$, because of the existence and global stability of $E_{n-1}$, we see that there exists an $\epsilon_{n}$ satisfying $0<\epsilon_{n}<\alpha_{n-1}$, such that (4.9) holds for $E_{n-2}$ if $\alpha_{n} \leq \epsilon_{n}$. We thus define $\alpha_{n}=\epsilon_{n}$.

Finally, because of (4.12), we see that (4.9) holds for $E_{n-1}$ as well. That is, we have found $\alpha_{i}>0, i=1, \ldots, n$, such that (4.9) holds for $E_{i}, i=0,1, \ldots, n-1$. By Lemma 4.3, we conclude that $\left(3.4^{\prime}\right),(2.2)-(2.3)$ is uniformly persistent.

REMARK 4.1. In theory, the discussion of this section can be applied to the full system (2.1) as well. The main difficulty is that we do not know how many spatially homogeneous steady states the system may have and where they may be located.

\section{Discussion}

It is not difficult to see that our approach can be applied to systems satisfying the food pyramid condition, with each trophic level consisting of several competing species. Of course, the analysis will become more complicated. 
Our main finding in this paper is that if the reaction system of the considered food chain has a globally asymptotically stable steady state, or the system possesses some kind of persistence, then the diffusive food chain is also likely to have the same properties, regardless of the magnitude of diffusion rates. In fact, we can even allow the diffusion rates to be zero for the lower trophic level species. This is in contrast to the situation when the reaction system has an unstable positive steady state. In [38], Mimura and Murray were able to show that when the diffusion of the prey is small compared with that of the predator, a predator-prey system (a food chain of length two) may exhibit, asymptotically in time, stable heterogeneity (patchiness) in a bounded domain with zero flux boundary conditions. The interested reader is referred to [39] for a systematic discussion of this phenomenon. However, it is well known that if diffusion rates are large, then a reaction-diffusion system has very similar qualitative properties to those possessed by its reaction system $[23,46]$. Our finding is also consistent with recent works in global stability of diffusive-delay Lotka-Volterra type systems [35,37], where the results also indicate that diffusion rates are not important in the qualitative analysis of the system.

For a delayed food chain with or without a diffusion effect, a detailed qualitative analysis seems to be difficult to reach. The main difficulty is that even the boundedness of solutions is not easy to establish, unless we assume that the system has some strong instant self-crowding effect. Nevertheless, we believe that these systems deserve future attention.

Finally, we would like to mention that the four-species simple food-chain model studied in [13] can be adapted to serve as a nice example for this paper. By combining this work with earlier results on global stability of predator-prey systems and studies of persistence in three-dimensional models (see, for example, $[7,14,15,32]$ ), we should be able to obtain more specific and sharper results for three- or four-species food chains with or without diffusion.

\section{Acknowledgement}

This research was partially supported by NSF Grant DMS-0077790.

\section{References}

[1] N. Alikakos, "An application of the invariance principle to reaction-diffusion equations", J. Diff. Equations 33 (1979) 301-325.

[2] J. R. Beddington and P. S. Hammond, "On the dynamics of host-parasite-hyperparasite interactions", J. Anim. Ecol. 46 (1977) 811-821.

[3] T. Burton and V. Hutson, "Repellers in systems with infinite delay", J. Math. Anal. Appl. 137 (1989) 240-263. 
[4] G. Butler, H. I. Freedman and P. Waltman, "Uniformly persistent systems", Proc. Amer. Math. Soc. 96 (1986) 425-430.

[5] G. Butler and P. Waltman, "Persistence in dynamical systems", J. Differential Equations 63 (1986) 255-263.

[6] T. J. Case and R. G. Casten, "Global stability and multiple domains of attraction in ecological systems", Amer. Nat. 113 (1979) 705-714.

[7] K.-S. Cheng, S.-B. Hsu and S.-S. Lin, "Some results on global stability of a predator-prey system", J. Math. Biol. 12 (1981) 115-126.

[8] M. Conrad, "Stability of foodwebs and its relation to species diversity", J. Theoret. Biol. 34 (1972) 325-335.

[9] D. L. DeAngelis, "Stability and connectance in food web models", Ecology 56 (1975) 238-243.

[10] D. L. DeAngelis and R. A. Goldstein, "Criteria that forbid a large, nonlinear food-web model from having more than one equilibrium point", Math. Biosci. 41 (1978) 81-90.

[11] S. R. Dunbar, K. P. Rybakowski and K. Schmitt, "Persistence in models of predator-prey populations with diffusion", J. Differential Equations 65 (1986) 117-138.

[12] H. I. Freedman, Deterministic mathematical models in population ecology (Marcel Dekker, New York, 1980).

[13] H. I. Freedman and J. W.-H. So, "Global stability and persistence of simple food chains", Math. Biosci. 76 (1985) 69-86.

[14] H. I. Freedman and P. Waltman, "Persistence in models of three interacting predator-prey populations", Math. Biosci. 68 (1984) 213-231.

[15] H. I. Freedman and P. Waltman, "Persistence in a model of three competitive populations", Math. Biosci. 73 (1985) 89-101.

[16] T. C. Gard, "Persistence in food webs: Holling-type food chains", Math. Biosci. 49 (1980) 61-67.

[17] T. C. Gard, "Persistence in food webs with general interactions", Math. Biosci. 51 (1980) 165-174.

[18] T. C. Gard, "Persistence for ecosystem microcosm models", Ecol. Model. 12 (1981) 221-229.

[19] T. C. Gard, "Top predator persistence in differential equation models of food chains: The effect of omnivory and external forcing of lower trophic levels", J. Math. Biol. 14 (1982) 285-299.

[20] T. C. Gard, "Uniform persistence in multispecies population models", Math. Biosci. 85 (1987) 93-104.

[21] T. C. Gard and T. G. Hallam, "Persistence in food webs-1. Lotka-Volterra food chains", Bull. Math. Biol. 41 (1979) 877-891.

[22] B.-S. Goh, "Global stability in a class of predator-prey models", Bull. Math. Biol. 40 (1978) 525-533.

[23] J. K. Hale, "Large diffusivity and asymptotic behavior in parabolic systems", J. Math. Anal. Appl. 118 (1986) $455-466$.

[24] J. K. Hale and P. Waltman, "Persistence in infinite-dimensional systems", SIAM J. Math. Anal. 20 (1989) 388-395.

[25] G. W. Harrison, "Global stability of food chains", Amer. Nat. 114 (1979) 455-457.

[26] A. Hastings, "Global stability in Lotka-Volterra systems with diffusion", J. Math. Biol. 6 (1978) 163-168.

[27] D. Henry, Geometric theory of semilinear parabolic equations, Lecture Notes 840 (1981).

[28] J. Hofbauer and K. Sigmund, The theory of evolution and dynamical systems (Cambridge University Press, 1988).

[29] V. Hutson and W. Moran, "Repellers in reaction-diffusion systems", Rocky Mountain J. Math. 17 (1987) 301-314.

[30] V. Hutson and K. Schmitt, "Permanence in dynamical systems", preprint.

[31] V. Hutson and T. Vickers, "A criterion for permanent coexistence of species, with an application to a two-prey one-predator system", Math. Biosci. 63 (1983) 253-269. 
[32] Y. Kaung, "Global stability of Gause-type predator-prey systems", J. Math. Biol. 28 (1990) 463474.

[33] Y. Kuang and H. I. Freedman, "Uniqueness of limit cycles in Gause-type models of predator-prey systems", Math. Biosci. 88 (1988) 67-84.

[34] Y. Kuang, R. H. Martin and H. L. Smith, "Global stability for infinite delay, dispersive LotkaVolterra systems; weakly interacting populations in nearly identical patches", J. Dynamics and Differential Equations 3 (1991) 339-360.

[35] Y. Kuang and H. L. Smith, "Global stability in diffusive delay Lotka-Volterra systems", Differential and Integral Equations 4 (1991) 117-128.

[36] Y. Kuang and H. L. Smith, "Global stability for infinite delay Lotka-Volterra type systems", J. Diff. Eqns. 103 (1992) 221-246.

[37] R. H. Martin and H. L. Smith, "Convergence in Lotka-Volterra systems with diffusion and delay", in Diff. Eqns with Appl. in Biology, Physics and Engineering (ed. J. A. Goldstein et al.), (Marcel Dekker, 1989).

[38] M. Mimura and J. D. Murray, “On a diffusive prey-predator model which exhibits patchiness", $J$. Theor. Biol. 75 (1978) 249-262.

[39] J. D. Murray, Mathematical biology, Biomathematics Text 19 (Springer, 1989).

[40] R. Redheffer, R. Redlinger and W. Walter, "A theorem of LaSalle-Lyapunov type for parabolic systems", SIAM J. Math. Anal. 19 (1982) 99-103.

[41] R. Redlinger, "Über die $C^{2}$-Kompaktheit der Bahn von Lösungen semilinearer parabolischer Systeme", Proc. Roy. Soc. Edinburgh, Sect. A 93 (1982) 99-103.

[42] R. Redlinger, "Compactness results for time-dependent parabolic systems", J. Differential Equations 64 (1986) 133-153.

[43] A. Rescigno and K. G. Jones, "The struggle for life: III. A predator-prey food chain", Bull. Math. Biophys. 34 (1972) 521-532.

[44] D. J. Rogers and M. P. Hassell, "General models for insect parasite and predator searching behaviour: Interference”, J. Anim. Ecol. 43 (1974) 239-253.

[45] P. T. Saunders and M. J. Bazin, "On the stability of food chains", J. Theoret. Biol. 52 (1975) 121-142.

[46] J. Smoller, Shock waves and reaction diffusion equations (Springer, New York, 1982).

[47] Y. Takeuchi and N. Adachi, "The existence of globally stable equilibria of ecosystems of the generalized Volterra type", J. Math. Biol. 10 (1980) 401-415.

[48] Y. Takeuchi, N. Adachi and H. Tokumaru, "The stability of generalized Volterra equations", J. Math. Anal. Appl. 62 (1978) 453-473.

[49] V. Volterra, "Variazionie fluttuazioni del numero d'individui in specie animali conviventi", Mem. Acad. Lincei. 2 (1926) 31-113. 